\title{
The rare splenic abscess
}

\author{
Elroy Weledji ${ }^{1}$ and Frank Zouna ${ }^{1}$ \\ ${ }^{1}$ University of Buea
}

December 22, 2021

\begin{abstract}
Although the spleen is frequently enlarged in association with systemic infection, splenic abscesses are rare. They result from direct or haematogenous spread, or when a haematoma becomes infected. We present a case of the rare splenic abscess in a low resource setting.
\end{abstract}

\section{Introduction}

Abscesses of the spleen are relatively uncommon with an incidence of $0.2 \%$ [1]. Splenic abscesses have been reported periodically since the time of Hippocrates who postulated that the condition may take one of the following three courses; (i) the patient might die, (ii) the abscess might heal or (iii) the abscess might become chronic and the patient might live with the disease. He was essentially correct because the aetiology varies $[1-4]$

\section{Case history/examination}

A 26-year- old African male farmer presented with a 3-month history of a rapidly progressing left hypochondrial mass. This was associated with abdominal pain and intermittent fever. He had no history of trauma, infective endocarditis nor tuberculosis. He was neither human immunodeficiency virus (HIV) positive nor diabetic. A full blood count revealed a low haemoglobin level $(\mathrm{Hb} 6.1 \mathrm{~g} / \mathrm{dl})$, a leucocytosis of $16.5 \times 10^{9} / \mathrm{l}$ , and, a normal platelet count of $307 \times 10^{9} / 1$. Physical examination demonstrated a tender splenomegaly extending to the umbilicus (Hackett's 4). There was no associated lymphadenopathy and cardiovascular examination was normal. The differential diagnosis would include space-occupying lesions or splenic masses due to trauma leading to haematoma or rupture, splenic abscess, tumours and cysts. An abdominal ultrasound scan suggested a splenic abscess. Following the transfusion of 2 units of whole blood, he underwent a difficult resection of a large necrotic fluid- filled spleen which was densely adherent to the tail of the pancreas, inferior surface of the liver and greater curve of the stomach (Fig 1). Macroscopically, the resected spleen was large, multiloculated with total parenchymal destruction (Fig 2). He made good recovery and was discharged a week later after receiving vaccines against the encapsulated bacterial organisms, streptocococcus pneumonia, haemophilus influenza andneissieria meningitides that may cause an overwhelming post splenectomy infection (OPSI).

\section{Discussion}

Splenic abscesses are commonly caused by septic emboli from complications of infective endocarditis in about $5 \%$ of these patients, with the pathogens being streptococcus or staphylococcus [1]. It carries a very high mortality of greater than $70 \%$ if the diagnosis is missed, but with appropriate treatment the mortality can be reduced to less than $1 \%$. With the availability of CT scan today the condition is rapidly diagnosed in addition to a potential treatment by aspiration [1-3]. The second common cause is a secondary infection of an infarcted spleen following trauma, interventional radiological embolization of splenic artery pseudoaneurysm complicating acute pancreatitis or a haemoglobinopathy such as sickle cell disease $[1,3,5]$. Other risk factors 
include immunocompromised states with $80 \%$ mortality, diabetes mellitus, illicit intravenous drug use in which splenic abscesses occur from a contiguous focus of infection. In these cases, the organisms commonly associated are polymicrobial (>50\%), aerobes, anaerobes, fungi (usually candida) [1-4]. Salmonella typhosa has been a well- documented cause in the sickling disorders [6]. Splenic abscesses can also be associated with parasitic infection of the spleen and miscellaneous rare organisms such as Burkholderia. Mycobacterium and Actinomycetes [3]. Spontaneous rupture has been reported in a number of conditions in which the spleen is enlarged which includes typhoid, malaria, leukaemia, Gaucher's disease, and polycythemia. These may be restricted to a subcapsular haematoma or there may be rupture into the peritoneal cavity which would be suggested by the symptoms of shock, left upper quadrant guarding and tenderness, pain referred to the left shoulder, and clinical and radiological evidence of bleeding [7]. The common symptoms and signs of splenic abscess include the triad of fever, left upper quadrant tenderness and leukocytosis as seen in this case $[1,3,7,8]$. Just as with splenic cysts the definitive treatment is splenectomy as most of the spleen is affected (fig 2) [9, 10]. In addition, pneumococcal, haemophilus influenza type b and meningococcal conjugate vaccinations against the life-time risk (0.1-0.5\%) but $50 \%$ mortality from a subsequent OPSI is required [10]. Percutaneous drainage is less likely to be successful in patients with multilocular abscesses, illdefined cavities with necrotic debris and thick viscous fluid. Mortality rates of greater than $50 \%$ is reported in patients managed with antibiotics only $[1,3]$. Unfortunately, microbiological culture and sensitivity of a pus sample was not available to this patient which may have given a clue to its aetiology.

\section{Declarations}

Consent for publication: Written informed consent from the patient was granted to write and publish the paper.

Competing interests: The author declares no competing interests.

Availability of data and materials: not applicable

Funding: None

Authors' contributions: EPW was the surgeon and main author, FZ contributed to the preoperative care and literature search.

\section{References}

1. Lee MC, Lee CM. Splenic abscess: An uncommon entity with potentially life- threatening evolution. Can J Infect Dis Med Microbiol 2018;2018:8610657

2. Divyashre S, Gupta N. Splenic abscesses in immunocompetent patients managed primarily without splenectomy: A series of 7 cases. Perm J.2017;21:16-139

3. Liu YH, Liu CP, Lee CM. Splenic abscesses at a tertiary medical centre in Northern Taiwan. J Microbiol Immunol Infect 2014;47(2):104-8

4. Pastakia B, Shawker TH, Thaler M, et al. Hepatosplenic candidiasis: wheels within wheels. Radiology 1988;166(2):417-22

5. Mansueto G, Cenzi D, D'Onofrio M, et al. Endovascular treatment of arterial bleeding in patients with pancreatitis. Pancreatology. 2007;7(4):360-9

6. Al-Salem AH. Splenic complications of sickle cell anaemia and the role of splenectomy. ISRN Haematol 2011;2011:864257

7. Pang W, Zhang T, Chen Y . Space-occupying benign lesions in spleen: experiences in a single institute. Paediatr Surg Int 2009;25(1):31-5

8. Liverani E, Colecchia A, Mazella G. Persistent fever and abdominal pain in a young woman with Budd-Chiari syndrome . Gastroenterology 2018;154(3):495-497

9. Tagaya A, Hamada K, kubota K. Laparoscopic splenectomy for recurrent splenic cyst after laparoscopic marsupialization. Surg laparosc endosc percutan Tech 2007;17(5):465-8

10. Weledji EP. Benefits and risks of splenectomy. Int J Surg (Lond) 2014;12(2): 113-119 Figures 


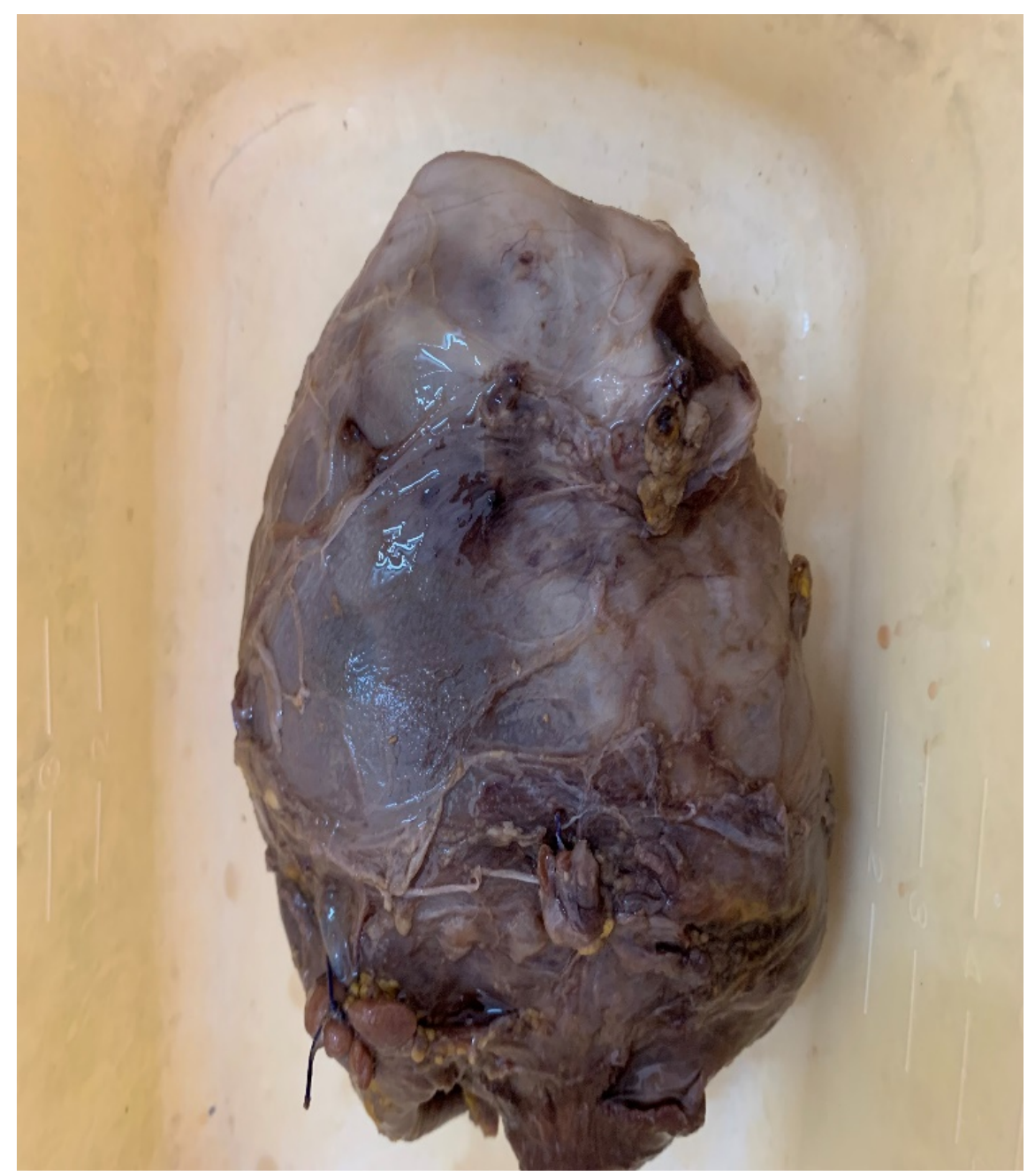

Figure 1'. Serosal surface of resected splenic abscess (formalin-fixed) 


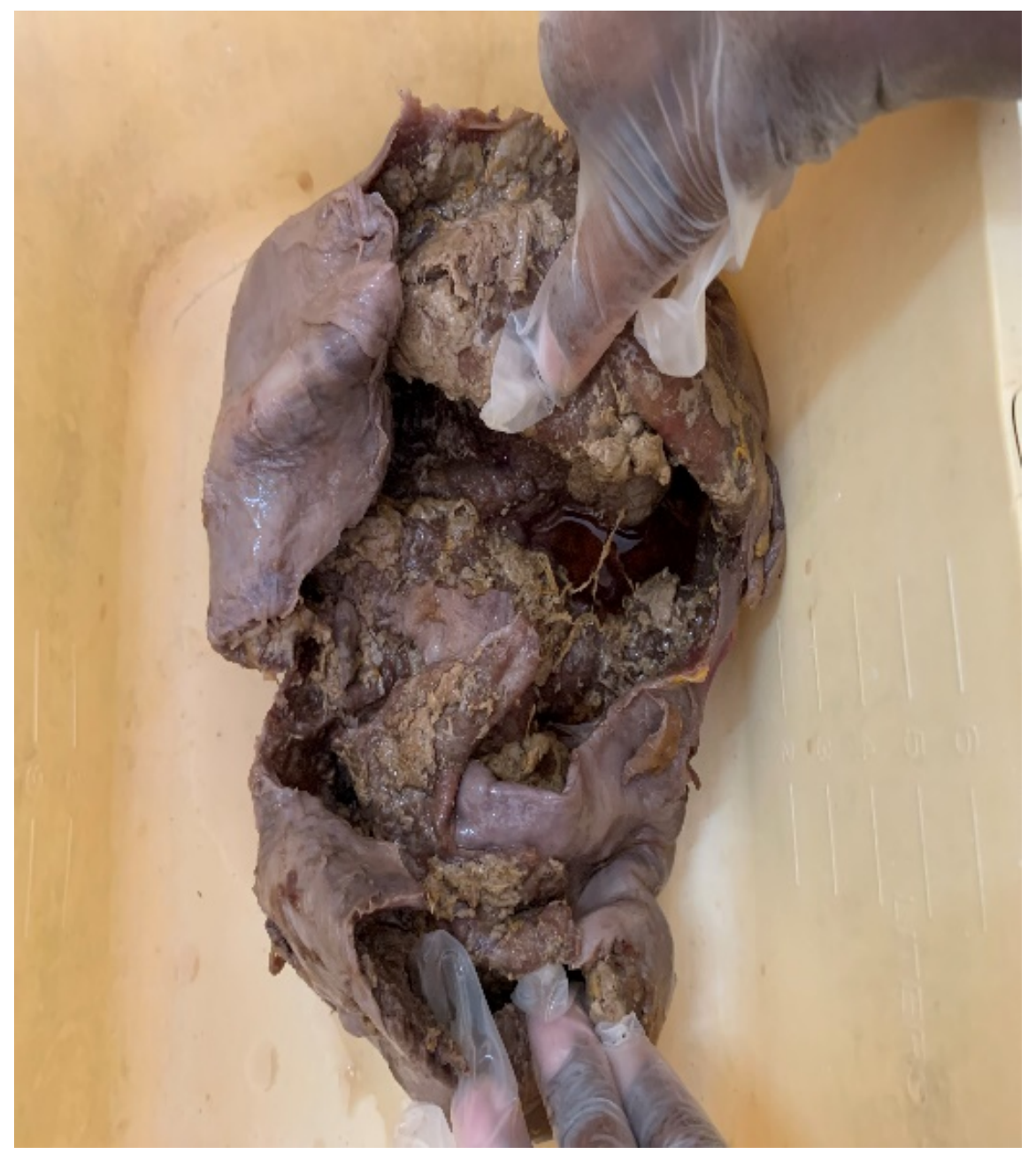

Figure 2: Multilocular cavities of a splenic abscess with complete loss of splenic parenchyma (formalin-fixed). 